\title{
VARREDURA ESPACIAL PARA IDENTIFICAÇÃO DE ÁREAS DE RISCO EPIDÊMICO E FATORES ASSOCIADOS A DENGUE: EXPERIÊNCIA EM BELO HORIZONTE, MINAS GERAIS
}

\section{SPATIAL ANALYSIS SCAN FOR IDENTIFICATION OF EPIDEMIC RISK AREAS AND FACTORS ASSOCIATED WITH DENGUE: EXPERIENCE IN BELO HORIZONTE, MINAS GERAIS}

\author{
Amanda Priscila de Santana Cabral Silva \\ Doutora em Saúde Pública - Fiocruz Pernambuco \\ Docente Adjunta do Centro Acadêmico de Vitória/ Universidade Federal de Pernambuco \\ amanda.cabral@ufpe.br \\ Dalva Maria de Assis \\ Mestre em Saúde Pública - Fiocruz Rio de Janeiro \\ Supervisora do Programa de Epidemiologia Aplicado aos Serviços do SUS - EpiSUS / Ministério da Saúde \\ dalva.assis@saude.gov.br \\ Lívia Carla Vinhal Frutuoso \\ Doutora em Epidemiologia - Universidade de Brasília \\ Tecnologista - Secretaria de Ciência Tecnologia e Insumos Estratégicos / Ministério da Saúde \\ livia.vinhal@saude.gov.br \\ Rodrigo Fabiano do Carmo Said \\ Especialista em Engenharia Sanitária - Universidade Federal de Minas Gerais \\ Consultor Nacional / Organização Pan Americana de Saúde \\ saidrod@paho.org
}

\section{RESUMO}

Objetivo: Apresentar a varredura espacial como instrumento para identificar clusters de dengue e fatores associados à epidemias em Belo Horizonte/MG. Métodos: Estudo ecológico, com casos de dengue notificados entre 2007 e 2010. Para identificação de clusters utilizou-se a estatística de varredura espacial Scan. A regressão logística investigou a associação entre clusters e o índice de infestação predial (IIP), proporção de imóveis verticalizados (PIV), densidade de ovos de Ae. aegypti (DO), cobertura de visitas de agentes de endemia e densidade populacional. Resultados: Ocorreram 76.592 casos de dengue e identificados 5 clusters por período: em 2007/2008 os agregados concentraram $30 \%$ da população e $67 \%$ dos casos; 2008/2009 agruparam 13\% da população e $60 \%$ dos casos e em $2009 / 2010$ concentraram $34 \%$ da população e $62 \%$ dos casos. Houve associação com IIP em 2007/2008 (OR=5,0; $p<0,01$ ) e alta DO em 2008/2009 (OR=8,4; $p=0,01)$ e 2009/2010 ( $O R=9,5 ; p<0,01)$. Conclusão: $A$ associação dos clusters aos indicadores operacionais revela a importância da utilização, por parte dos serviços de saúde, de estratégias de monitoramento entomológico que, associadas a varredura especial, podem contribuir no fortalecimento da vigilância territorializada da dengue para a determinação de áreas de risco e o planejamento de intervenções mais oportunas.

Palavras-chave: Arbovirose. Análise especial. Vigilância epidemiológica.

\begin{abstract}
Objective: To present spatial scanning as an instrument to identify clusters of dengue and factors associated with epidemics in Belo Horizonte / MG. Methods: Ecological study, with dengue cases notified between 2007 and 2010. For the identification of clusters, the spatial scanning statistic Scan was used. Logistic regression investigated the association between clusters and the building infestation index (IIP), proportion of verticalized buildings (PIV),
\end{abstract}

Recebido em: 11/09/2020

Aceito para publicação em: 21/01/2021. 
Varredura espacial para identificação de áreas de risco epidêmico e fatores associados a dengue: experiência em Belo Horizonte, Minas Gerais
Amanda Priscila de Santana Cabral Silva

Dalva Maria de Assis

Lívia Carla Vinhal Frutuoso

Rodrigo Fabiano do Carmo Said

density of Aedes Aegypti (DO), coverage of visits by endemic agents and population density. Results: There were 76,592 dengue cases and five clusters were identified per period: in $2007 / 2008$ the aggregates concentrated $30 \%$ of the population and $67 \%$ of the cases; $2008 / 2009$ grouped $13 \%$ of the population and $60 \%$ of cases and in $2009 / 2010$ concentrated $34 \%$ of the population and $62 \%$ of cases. There was an association with PII in $2007 / 2008(O R=5.0 ; p<0.01)$ and high $O D$ in $2008 / 2009(O R=8.4 ; p=0.01)$ and 2009/2010 (OR = 9,5; $p<0.01)$. Conclusion: Spatial scanning can be useful to strengthen territorialized surveillance of dengue by allowing identification of priority areas for effective disease control.

Keyword: Dengue. Spatial analysis. Epidemiological surveillance.

\section{INTRODUÇÃO}

Dengue é uma doença febril aguda que pode se apresentar nas formas de uma infecção inaparente até quadros graves e hemorrágicos podendo levar a morte. É uma das mais importantes arboviroses que afeta o ser humano, ocorrendo e disseminando-se principalmente nos países tropicais, onde as condições ambientais favorecem o desenvolvimento do vetor (VERONESI, 2015).

O Brasil é o país das Américas mais afetado em número de casos de dengue, concentrando aproximadamente $70 \%$ dos casos notificados (World Health Organization, 2009). No período de 2003 a maio de 2019, foram notificados mais de 11 milhões de casos prováveis de dengue no país, com o destaque de cinco anos epidêmicos (2008, 2010, 2013, 2015 e 2016), todos relacionadas a introdução / reintrodução de novos sorotipos (SOUZA ET AL, 2019).

Diversos fatores são condicionantes da expansão da dengue no Brasil e no mundo, como por exemplo, o crescimento desordenado das cidades com importantes lacunas no setor de infraestrutura, a rápida urbanização, a falta de saneamento básico, o aumento da circulação de pessoas, a existência de vários sorotipos distintos, as condições climáticas favoráveis e o difícil controle do vetor (MARQUES, 2020).

Com a ausência de uma vacina para combate a doença, as medidas de controle englobam ações das vigilâncias epidemiológicas e entomológicas. A primeira visa acompanhar a evolução temporal da incidência de casos em determinado espaço geográfico, identificar mudanças no padrão de circulação viral e no perfil dos casos, além disso, a vigilância epidemiológica organiza discussões conjuntas com equipes de controle de vetores e de assistência de maneira a orientar a organização dos serviços de saúde e as atividades de controle vetorial. A vigilância entomológica atua diretamente nas ações de controle vetorial. Geralmente essas atividades são realizadas por ciclos de trabalho, com periodicidade bimestral (MINISTÉRIO DA SAÚDE, 2009).

Embora tenha ocorrido o aumento nas notificações nos últimos anos, o ano de 2010 foi histórico para a Vigilância Epidemiológica da dengue no Brasil, quando foi contabilizado cerca de 1 milhão de notificações pela doença. Somente entre as semanas epidemiológicas 1 a 26 de 2010 o país registrou no Sistema Nacional de Agravos de Notificação (Sinan) 942.153 casos suspeitos de dengue, dos quais 182.789 foram em Minas Gerais. Belo Horizonte concentrou 48.906 casos, sendo o município com maior número de notificações na época (MINISTÉRIO DA SAÚDE, 2010).

O uso exclusivo das taxas de incidência para avaliar a distribuição geográfica de doenças pode produzir resultados limitados. A utilização de Sistemas de Informações Geográficas (SIG) podem contribuir como um instrumento de prevenção da transmissão da dengue, por localizar com maior precisão os casos da doença e a presença do vetor (PAVARINI, 2008).

Dessa forma, o objetivo desse estudo é demonstrar a aplicabilidade da técnica de varredura espacial como instrumento para identificar clusters de dengue e fatores associados a epidemias em Belo Horizonte, Minas Gerais. 
Varredura espacial para identificação de áreas de risco epidêmico e fatores associados a dengue: experiência em Belo Horizonte, Minas Gerais
Amanda Priscila de Santana Cabral Silva

Dalva Maria de Assis

Lívia Carla Vinhal Frutuoso

Rodrigo Fabiano do Carmo Said

\section{MÉTODOS}

Trata-se de um estudo ecológico de agregados populacionais, tendo como área de estudo Belo Horizonte, Minas Gerais. O município apresenta extensão territorial de $331,4 \mathrm{Km}^{2}$ e para o ano de de 2010 contava com 2.375.151 habitantes, caracterizando uma densidade demográfica de 7.167,02 hab/ $\mathrm{Km}^{2}$ (INSTITUTO BRASILEIRO DE GEOGRAFIA E ESTATÍSTICA, 2010).

O município esta dividido em nove distritos sanitários e 147 áreas de abrangência $(A B)$ dos centros de saúde (SOUZA ET AL, 2016), as quais corresponderam à unidade de análise deste estudo. $A$ AB tem território estabelecido com base em setores censitários contíguos, definidos pelo IBGE, sendo a base de organização da rede municipal de saúde. A equipe de controle de zoonoses está inclusa nas equipes dos centros de saúde de Belo Horizonte (SOUZA ET AL, 2016).

Foram utilizados três períodos de estudo: 01 de setembro de 2007 a 31 de agosto de 2008 (2007/2008), 01 de setembro de 2008 a 31 de agosto de 2009 (2008/2009) e 01 de setembro de 2009 a 31 de agosto de 2010 (2009/2010). O período entre setembro de um ano a agosto do ano seguinte representa a sazonalidade da doença, pois em geral, os meses de agosto e setembro apresentam os menores valores de incidência em relação aos demais meses do ano, enquanto entre janeiro e abril apresentam as maiores incidências.

A análise de dados foi composta por três etapas, dispostas a seguir:

$1^{\text {a }}$ etapa - identificação da existência de epidemia, por meio de diagramas de controle. Utilizou-se como série histórica os casos notificados por semana epidemiológica, a partir do ano 2000; foram excluídos os anos epidêmicos e calculadas a média móvel e os desvios-padrão das médias móveis da distribuição mensal, utilizando-se um período de cinco semanas (a semana de interesse acrescida de duas semanas anteriores e duas semanas posteriores) e das incidências registradas no período selecionado. O limite máximo esperado corresponde a média móvel somada a 1,96 desvios padrão. $O$ limite médio da doença corresponde à média móvel. Os valores do ano em questão compreendidos abaixo do limite superior correspondem ao nível endêmico da doença. Se estiverem acima, o período é classificado como epidêmico.

As incidências de dengue por $A B$ no período de estudo foram calculadas dividindo-se o número de casos novos pela estimativa da população base do período estudado da respectiva $A B$. Os resultados foram multiplicados por 100.000 e agrupados por quartil, possibilitando a comparação dos três períodos estudados.

$2^{a}$ etapa - Confirmação de aglomerados espaciais de casos de dengue, considerando como hipótese nula que, os casos totais do município são distribuídos de forma homogênea em todas as $A B$, sendo proporcional à população residente. A hipótese alternativa foi a de que o número total de casos se distribui de forma heterogênea, gerando aglomerados espaciais de casos (KULLDORFF, 2015).

O modelo probabilístico adotado foi do tipo Poisson, assumindo que a distribuição do número de casos no município é homogênea. $O$ ajuste da ausência de homogeneidade na população é atingido condicionando-se o número total de casos observados para calcular o número esperado de casos para cada $A B$.

Foi adotada a estatística de varredura espacial, por meio do SatScan ${ }^{\circledR}$, que cria janelas com base circular correspondente a uma área geográfica; os tamanhos são variáveis e permite que seu centro se mova na superfície do mapa, onde para cada posição e tamanho, a janela inclua um conjunto diferente de vizinhos próximos. Se a janela incluir o centróide de um vizinho, então toda a $A B$ é considerada inclusa. Como a janela se move passando por todos os centróides, seu raio varia continuamente de zero ao raio máximo, que neste estudo nunca inclui mais do que $25 \%$ da população total. A função de probabilidade é maximizada sobre todas as janelas, identificando a janela que constitui o agrupamento mais provável. A razão de probabilidade para esta janela é anotada e constitui o teste estatístico da razão de probabilidade máxima (KULLDORFF, 2015).

Sua distribuição sob a hipótese nula e seu valor de $p$ simulado correspondente é obtido pela repetição do mesmo exercício analítico, num grande número de réplicas aleatórias (999) do conjunto de dados gerados sob a hipótese nula, numa simulação do tipo Monte Carlo (KULLDORFF, 2015). A hipótese de nulidade foi rejeitada quando $p<0,05$ para o aglomerado mais provável, que em outras palavras significa que naquela região observou-se que o risco de seus residentes adoecerem por dengue é maior do que a risco esperado na mesma região.

DOI: http://dx.doi.org/10.14393/Hygeia17057163 $\quad$ Hygeia $\quad$ v.17 $\quad$ p. 14-25, 2021 página 16


Varredura espacial para identificação de áreas de risco epidêmico e fatores associados a dengue: experiência em Belo Horizonte, Minas Gerais
Amanda Priscila de Santana Cabral Silva

Dalva Maria de Assis

Lívia Carla Vinhal Frutuoso

Rodrigo Fabiano do Carmo Said

$3^{a}$ etapa - Identificação, por meio de regressão logística, de fatores associados à ocorrência de surtos. Com a disponibilidade de dados do controle vetorial, buscou-se identificar associações entre a ocorrência de dengue nos agrupamentos de risco para surtos, identificados na etapa anterior, e os seguintes fatores:

- Índice de infestação predial (IIP): proporção do número de imóveis com larvas e/ou pupas do Ae. Aegypti em relação ao total de imóveis pesquisados. Considerou-se a leitura do índice que antecedeu o período epidêmico registrado.

- Índice de densidade de ovos: razão entre o número de ovos de Ae. aegypti e o número de armadilhas (ovitrampas) para coleta de ovos positivos (Ministério da Saúde, 2009b). Foi calculada a média das leituras do índice em cada período de estudo.

- Percentual de imóveis verticalizados: estudos sugerem que altos percentuais desta condição seria um fator de proteção contra ocorrência de casos de dengue, uma vez que a baixa altitude e menor circulação de massas de ar, comuns em áreas horizontais podem favorecer a domiciliação do vetor (Cunha, 2008).

- Cobertura de visitas dos agentes de controle de zoonozes: se refere à relação entre os imóveis que foram efetivamente vistoriados e tratados para o controle vetorial da dengue e o total de imóveis que deveriam ser vistoriados e tratados.

- Densidade populacional de área ocupada: razão entre o número de habitantes por metro quadrado ocupado (excluindo áreas não habitadas).

Para identificar a associação entre as variáveis independentes e a ocorrência de aglomerados espaciais, utilizou-se a análise univariada entre a variável dependente e as variáveis independentes para determinar a magnitude e a significância estatística das associações, pré-selecionando aquelas com valor $p<0,20$. $O$ modelo final foi composto por variáveis com $p \leq 0,05$.

No plano de análise, o índice de infestação predial foi categorizado segundo parâmetros utilizados pelo Ministério da Saúde, onde IIP < 1\% = satisfatório (referência); entre 1 e 3,9\%=alerta; $>3,9 \%=$ risco (Ministério da Saúde, 2009b). As demais variáveis independentes foram categorizados por quartis, sendo o quartil 1 utilizado como a referência. Para a variável dependente, as $A B$ que formaram os agrupamentos de risco para surtos foram categorizadas como 1 (um); as $A B$ não significativas na categoria 0 (zero).

Os softwares utilizados foram o Terraview 4.2.0, Satscan 8.2.1, Epiinfo 3.5.1 e Microsoft Exce ${ }^{\circledR}$. A Secretaria Municipal de Saúde de Belo Horizonte, sob solicitação oficial, disponibilizou para este estudo o banco de dados de dengue do Sistema Nacional de Agravos de Notificação (Sinan) e do Sistema de Vigilância Municipal com os endereços de residência dos casos notificados georreferenciados não nominais. Também foi cedida a estimativa populacional, base cartográfica e dados do controle vetorial.

O estudo foi aprovado pelo Comitê de Ética em Pesquisa da Secretaria Municipal de Saúde de Belo Horizonte sob o número do certificado de apresentação para apreciação ética (CAAE) 0056.0.410.000-10A

\section{RESULTADOS}

No município de Belo Horizonte, entre 1 de setembro de 2007 e 31 de agosto de 2010, foram notificados 76.592 casos de dengue, sendo 12.484 entre 2007 e 2008, 12.425 entre 2008 e 2009 e 51.683 casos entre 2009 e 2010. Nos três anos estudados a mediana de idade variou de 30 a 31 anos e o sexo feminino foi predominante, variando de 54 a 56\%. Do total de notificações, $287(0,4 \%)$ não foram georreferenciadas devido a possíveis inconsistências campo endereço presente na ficha de notificação do Sinan.

Em todo o período analisado foram observados comportamentos epidêmicos. Embora ocorram em época do ano semelhante, as epidemias contemplam diferentes números de semanas epidemiológicas: enquanto em 2007/2008 a epidemia se estendeu da SE 12 a 27/2008, em 2008/2009 ocorreu entre a SE 1 a 17/2009, sendo a de 2009/2010 a mais longa, entre a SE 48/2009 a 27/2010 (Figura 1). Considerando a distribuição espacial da incidência, segundo área de abrangência de residência, levanta-se a hipótese da existência de aglomerados espaciais (Figura 1). 
Varredura espacial para identificação de áreas de risco epidêmico e fatores associados a dengue: experiência em Belo Horizonte, Minas Gerais
Amanda Priscila de Santana Cabral Silva

Dalva Maria de Assis

Lívia Carla Vinhal Frutuoso Rodrigo Fabiano do Carmo Said

Figura 1 - Diagrama de Controle e incidência de dengue segundo área de abrangência de residência em Belo Horizonte, MG. (A) Setembro/2007 a agosto/2008; (B) Setembro/2008 a agosto/2009; (C)

Setembro/2009 a agosto/2010.

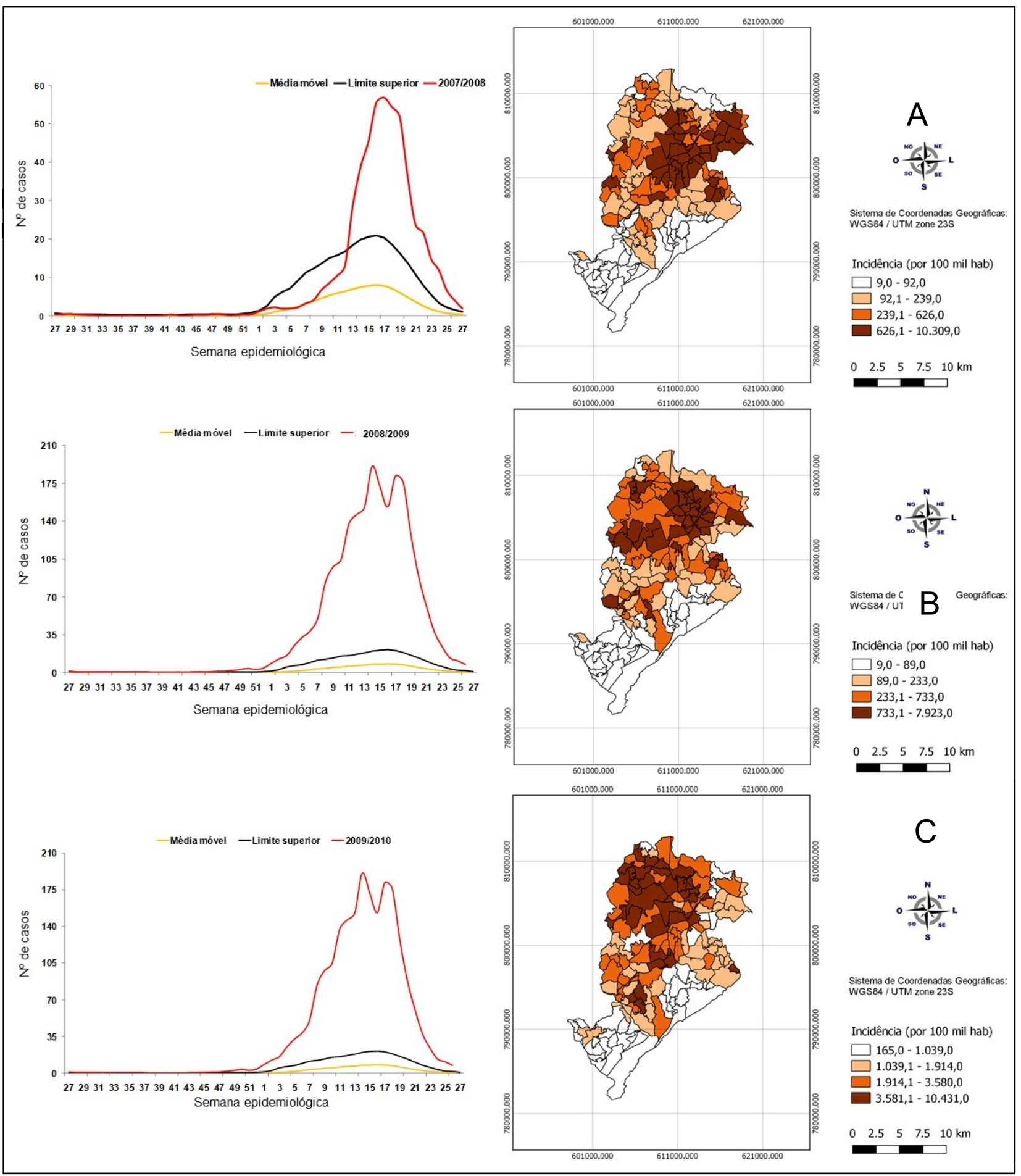

Fonte: Elaborado pelos autores 
Varredura espacial para identificação de áreas de risco epidêmico e fatores associados a dengue: experiência em Belo Horizonte, Minas Gerais
Amanda Priscila de Santana Cabral Silva

Dalva Maria de Assis

Lívia Carla Vinhal Frutuoso Rodrigo Fabiano do Carmo Said

Com a aplicação da estatística de varredura identificou-se aglomerados espaciais em cada um dos períodos estudados (Figura 2). Entre 2007/2008, 54 AB formaram 5 clusters estatisticamente significantes; juntos concentraram $30,4 \%$ da população e responderam por $67,3 \%$ dos casos. Neste período destacam-se os residentes do Cluster 1, todos localizados no Distrito Nordeste, que tiveram um risco 15 vezes maior de terem dengue comparado aos residentes das demais $A B$ (Tabela 1 ).

Em 2008/2009, 25 AB formaram 6 clusters estatisticamente significantes, que correspondeu a 13,3\% dos residentes de Belo Horizonte e concentraram $58,6 \%$ dos casos. O Cluster 1, formado por AB localizadas nos Distritos Norte e Nordeste, concentram $8,2 \%$ da população e $48,9 \%$ do total de casos do município. Apenas neste cluster, o risco de seus residentes terem tido dengue, foi 11 vezes maior comparado aos não residentes (Tabela 1).

No período 2009/2010, 55 AB formaram 6 clusters significantes, compostos por 33,7\% da população e por $62 \%$ dos casos. Neste ano, o Cluster 1 concentrou 42 AB localizadas nos Distritos Norte, Venda Nova e Pampulha, representando $24,7 \%$ dos residentes do município e com $48,0 \%$ dos casos de dengue. Seus residentes tiveram um risco 3 vezes maior de terem dengue comparados aos residentes das demais regiões (Tabela 1).

Figura 2 - Aglomerados espaciais de risco de dengue em Belo Horizonte, MG. A. setembro/2007 a agosto/2008; B. setembro/2008 a agosto/2009; C. setembro/2009 a agosto/2010.

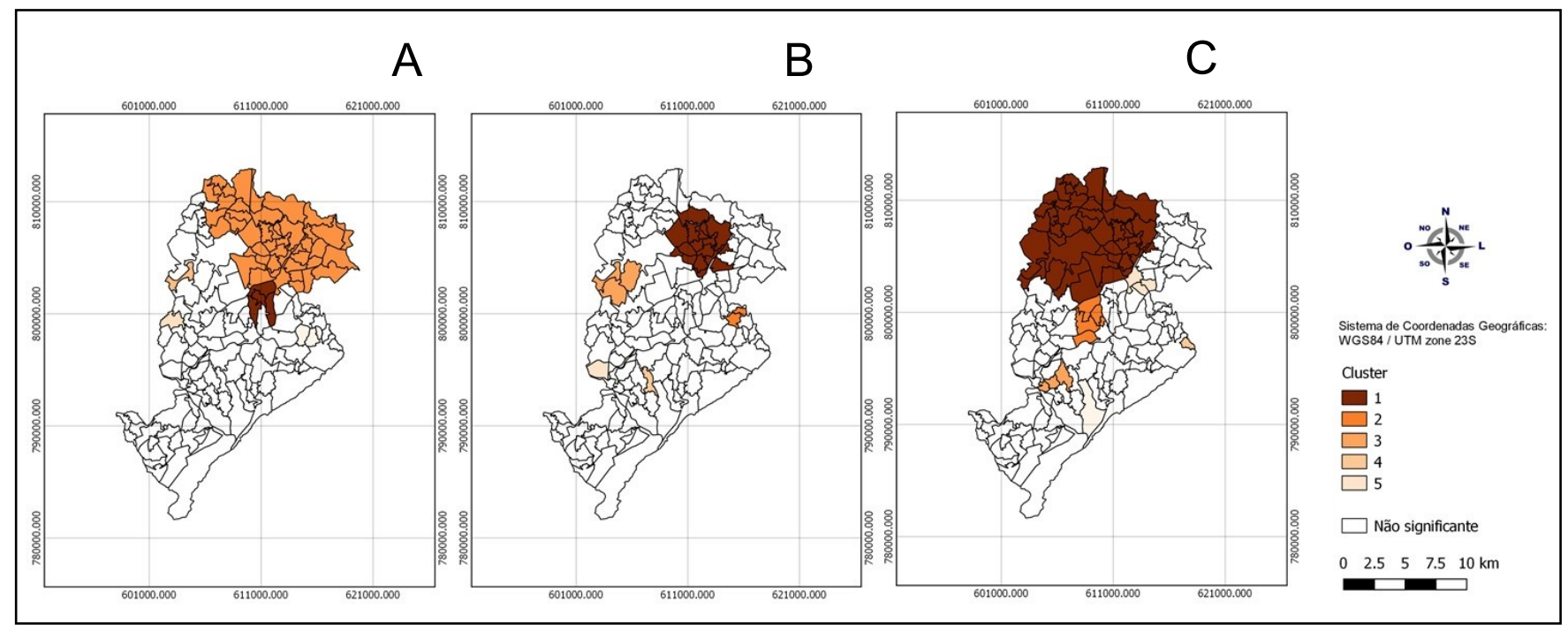

Fonte: Elaborado pelos autores. 
Varredura espacial para identificação de áreas de risco epidêmico e fatores associados a dengue: experiência em Belo Horizonte, Minas Gerais
Amanda Priscila de Santana Cabral Silva

Dalva Maria de Assis

Lívia Carla Vinhal Frutuoso Rodrigo Fabiano do Carmo Said

Tabela 1 - Características dos aglomerados espaciais de casos de dengue. Belo Horizonte, MG. Setembro de 2007 a agosto de 2010.

\begin{tabular}{lrrrrrrrr}
\hline Período & Cluster & $\begin{array}{c}\text { População } \\
\text { Exposta (N) }\end{array}$ & $\begin{array}{c}\text { População } \\
\text { total }\end{array}$ & $\begin{array}{c}\text { Casos } \\
\text { Esperados } \\
(\mathrm{N})\end{array}$ & $\begin{array}{c}\text { Casos } \\
\text { Observados } \\
(\mathrm{N})\end{array}$ & $\begin{array}{c}\text { \% casos do } \\
\text { município }\end{array}$ & RR $^{*}$ & IC (95\%) \\
& 1 & 69.998 & 3,1 & 389 & 4.050 & 32,5 & 10,4 & $9,4-11,6$ \\
& 2 & 525.246 & 23,5 & 2.918 & 3.680 & 29,6 & 1,3 & $1,2-1,3$ \\
Set/2007 - & 3 & 10.773 & 0,5 & 60 & 138 & 1,1 & 2,3 & $1,3-3,1$ \\
ago/2008 & 4 & 22.591 & 1,0 & 125 & 225 & 1,8 & 1,8 & $1,5-2,2$ \\
& 5 & 50.963 & 2,3 & 364 & 283 & 2,3 & 1,3 & $1,1-1,5$ \\
\hline & 1 & 183.947 & 8,2 & 1.018 & 6.057 & 48,9 & 5,9 & $5,6-6,4$ \\
Set/2008 - & 2 & 24.468 & 1,1 & 135 & 340 & 2,7 & 2,5 & $2,1-3,1$ \\
ago/2009 & 3 & 41.389 & 1,8 & 229 & 371 & 3,0 & 1,6 & $1,4-1,9$ \\
& 4 & 24.650 & 1,1 & 136 & 238 & 1,9 & 1,8 & $1,4-2,2$ \\
& 5 & 15.151 & 0,7 & 84 & 162 & 1,3 & 1,9 & $1,5-2,5$ \\
\hline & 1 & 540.955 & 24,7 & 12.444 & 24.724 & 48,0 & 2,0 & $1,9-2,0$ \\
Set/2009- & 2 & 89.342 & 4,0 & 2.055 & 3.379 & 6,6 & 1,6 & $1,5-1,7$ \\
ago/2010 & 3 & 50.686 & 2,3 & 1.166 & 1.847 & 3,6 & 1,6 & $1,5-1,7$ \\
& 4 & 11.297 & 0,5 & 260 & 474 & 0,9 & 1,8 & $1,6-2,1$ \\
& 5 & 32.210 & 1,4 & 741 & 944 & 1,8 & 1,3 & $1,2-1,4$
\end{tabular}

${ }^{*} \mathrm{p}-$ valor $<0,001$

Fonte: Elaborado pelos autores

As associações entre clusters e variáveis independentes propostas neste estudo são apresentadas na tabela 2, enquanto a tabela 3 mostra o modelo final das associações. Em 2007/2008, os clusters tiveram uma razão de chance 5 vezes maior de terem um índice de infestação predial > 3\% comparada as demais regiões; também observou-se que os clusters tiveram $90 \%$ menos chance de apresentarem imóveis verticalizados em seu território comparada as áreas não significantes. A densidade de ovos captadas em ovitrampas foi a única associação com clusters identificada em 2008/2009. Os clusters tiveram 8 vezes mais chance de terem uma alta densidade de ovos ( $4^{\circ}$ quartil) comparada às demais áreas. Por fim, em 2009/2010, a chance do cluster ter uma densidade de ovos no $4^{\circ}$ quartil é 9,5 vezes maior comparada às demais regiões; nesse período também se observou que os clusters tiveram $70 \%$ menos chance de apresentarem imóveis verticalizados em seu território comparada as áreas de abrangência não significantes. 
Varredura espacial para identificação de áreas de risco epidêmico e fatores associados a dengue: experiência em Belo Horizonte, Minas Gerais
Amanda Priscila de Santana Cabral Silva

Dalva Maria de Assis

Lívia Carla Vinhal Frutuoso Rodrigo Fabiano do Carmo Said

Tabela 2 - Associação dos aglomerados espaciais para ocorrência de dengue identificados com variáveis independentes. Belo Horizonte, MG, setembro de 2007 a agosto de 2010.

\begin{tabular}{|c|c|c|c|c|c|c|c|c|c|c|c|c|}
\hline \multirow{3}{*}{$\begin{array}{l}\text { Variáveis } \\
\text { Cobertura de } \\
\text { visitas }\end{array}$} & \multicolumn{4}{|c|}{ Set/2007 a Ago/2008 } & \multicolumn{4}{|c|}{ Set/2008 a Ago/2009 } & \multicolumn{4}{|c|}{ Set/2009 a Ago/2010 } \\
\hline & Intervalo & OR & IC (95\%) & $\mathrm{p}$ & Intervalo & OR & IC (95\%) & $\mathrm{p}$ & Intervalo & OR & IC (95\%) & $\mathrm{p}$ \\
\hline & & & & & & & & & & & & \\
\hline \multirow{2}{*}{$\begin{array}{l}1 \text { quartil } \\
2 \text { quartil }\end{array}$} & $\leq 55,7$ & 1,0 & - & - & $\leq 52,8$ & 1,0 & - & - & $\leq 53,2$ & 1,0 & - & - \\
\hline & $55,8-61,2$ & 2,8 & $1,0-7,6$ & 0,05 & $52,9-57,9$ & 1,2 & $0,3-4,5$ & 0,74 & $53,3-58,7$ & 0,4 & $0,1-1,0$ & 0,05 \\
\hline 3 quartil & $61,3-70,5$ & 1,2 & $0,4-3,6$ & 0,73 & $58,0-67,4$ & 1,3 & $0,4-4,6$ & 0,71 & $58,8-65,9$ & 0,7 & $0,3-1,7$ & 0,38 \\
\hline 4 quartil & $\geq 70,5$ & $\underline{4,8}$ & $\underline{1,7-13,2}$ & $\underline{0,01}$ & $\geq 67,5$ & 1,8 & $0,5-6,0$ & 0,36 & $>65,9$ & 1,4 & $0,6-3,5$ & 0,48 \\
\hline \multicolumn{13}{|l|}{$\begin{array}{l}\text { Dens. Pop. } \\
\text { área ocupada } \\
\left(\mathrm{hab} / \mathrm{km}^{2}\right)\end{array}$} \\
\hline \multirow{2}{*}{$\begin{array}{l}1 \text { quartil } \\
2 \text { quartil }\end{array}$} & $\leq 6.826$ & 1,0 & - & - & $\leq 6.826$ & 1,0 & - & - & $\leq 6.826$ & 1,0 & - & - \\
\hline & $\begin{array}{c}6.827- \\
9.727\end{array}$ & 1,2 & $0,5-3,2$ & 0,60 & $\begin{array}{c}6.827- \\
9.727\end{array}$ & 0,7 & $0,2-2,3$ & 0,53 & $\begin{array}{c}6.827- \\
9.727\end{array}$ & 0,9 & $0,4-2,3$ & 0,81 \\
\hline 3 quartil & $\begin{array}{l}9.728- \\
12.875\end{array}$ & 0,9 & $0,6-2,4$ & 0,90 & $\begin{array}{l}9.728- \\
12.875\end{array}$ & 0,9 & $0,3-2,9$ & 0,80 & $\begin{array}{l}9.728- \\
12.875\end{array}$ & 0,8 & $0,3-2,1$ & 0,70 \\
\hline 4 quartil & $>12.875$ & 0,6 & $0,3-1,8$ & 0,50 & $>12.875$ & 1,0 & $0,3-3,2$ & 1,00 & $>12.875$ & 0,8 & $0,3-2,0$ & 0,63 \\
\hline \multicolumn{13}{|l|}{$\begin{array}{l}\text { Índice de } \\
\text { infestação } \\
\text { predial }\end{array}$} \\
\hline \multirow{2}{*}{$\begin{array}{l}1 \text { quartil } \\
2 \text { quartil }\end{array}$} & $<1,0$ & 1,0 & - & - & $<1,0$ & 1,0 & - & - & $<1,0$ & 1,0 & - & - \\
\hline & $1,0-2,9$ & $\underline{3,4}$ & $\underline{1,1-10,7}$ & $\underline{0,04}$ & $1,0-2,9$ & 1,0 & $0,4-2,4$ & 0,99 & $1,0-2,9$ & 0,9 & $0,2-4,0$ & 0,90 \\
\hline 3 quartil & $\geq 3,0$ & $\underline{4,8}$ & $\underline{1,3-18,5}$ & $\underline{0,02}$ & $\geq 3,0$ & 0,0 & $0,0-1,0$ & 0,97 & $\geq 3,0$ & 1,3 & $0,3-6,1$ & 0,77 \\
\hline \multicolumn{13}{|l|}{$\begin{array}{l}\text { Média da } \\
\text { densidade de } \\
\text { ovos de A. } \\
\text { Aegytpi }\end{array}$} \\
\hline \multirow{2}{*}{$\begin{array}{l}1 \text { quartil } \\
2 \text { quartil }\end{array}$} & $\leq 15,4$ & 1,0 & - & - & $\leq 18,5$ & 1,0 & - & - & $\leq 18,7$ & 1,0 & - & - \\
\hline & $15,5-20,6$ & 0,8 & $0,3-2,2$ & 0,68 & $18,6-24,2$ & 2,7 & $0,5-15,1$ & 0,25 & $\begin{array}{c}18,8- \\
27,5\end{array}$ & 0,8 & $0,2-2,8$ & 0,76 \\
\hline \multirow{2}{*}{$\begin{array}{l}3 \text { quartil } \\
4 \text { quartil }\end{array}$} & $20,7-27,0$ & 0,9 & $0,3-2,3$ & 0,80 & $24,3-32,1$ & 3,5 & $0,7-18,6$ & 0,14 & $27,6-39,0$ & $\underline{3,4}$ & $\underline{1,2-9,8}$ & $\underline{0,02}$ \\
\hline & $>27,0$ & 2,0 & $0,8-5,1$ & 0,14 & $>32,1$ & $\underline{8,4}$ & $\underline{1,7-40,8}$ & $\underline{0,01}$ & $>39,0$ & $\underline{10,1}$ & $\underline{3,4-29,9}$ & $\underline{0,01}$ \\
\hline \multicolumn{13}{|l|}{$\begin{array}{l}\% \text { imóveis } \\
\text { verticalizados }\end{array}$} \\
\hline \multirow{2}{*}{$\begin{array}{l}1 \text { quartil } \\
2 \text { quartil }\end{array}$} & 0,0 & 1,0 & - & - & 0,0 & 1,0 & - & - & 0,0 & 1,0 & - & - \\
\hline & $0-0,2$ & 0,5 & $0,2-1,2$ & 0,10 & $0-0,2$ & 0,4 & $0,1-1,4$ & 0,16 & $0-0,2$ & 0,6 & $0,2-1,4$ & 0,24 \\
\hline \multirow{2}{*}{$\begin{array}{l}3 \text { quartil } \\
4 \text { quartil }\end{array}$} & $0,3-2,4$ & $\underline{0,2}$ & $\underline{0,1-0,6}$ & $\underline{0,01}$ & $0,3-2,4$ & 0,5 & $0,2-1,7$ & 0,29 & $0,3-2,4$ & 0,5 & $0,2-1,3$ & 0,16 \\
\hline & $>2,4$ & $\underline{0,1}$ & $\underline{0,0-0,3}$ & $\underline{0,00}$ & $>2,4$ & 0,3 & $0,1-1,2$ & 0,08 & $>2,4$ & $\underline{0,2}$ & $\underline{0,1-0,7}$ & $\underline{0,01}$ \\
\hline
\end{tabular}

Fonte: Elaborado pelos autores 
Varredura espacial para identificação de áreas de risco epidêmico e fatores associados a dengue: experiência em Belo Horizonte, Minas Gerais
Amanda Priscila de Santana Cabral Silva

Dalva Maria de Assis

Lívia Carla Vinhal Frutuoso Rodrigo Fabiano do Carmo Said

Tabela 3 - Modelo final de regressão logística da associação entre aglomerados espaciais para dengue e variáveis independentes associadas. Belo Horizonte, setembro de 2007 a agosto de 2010.

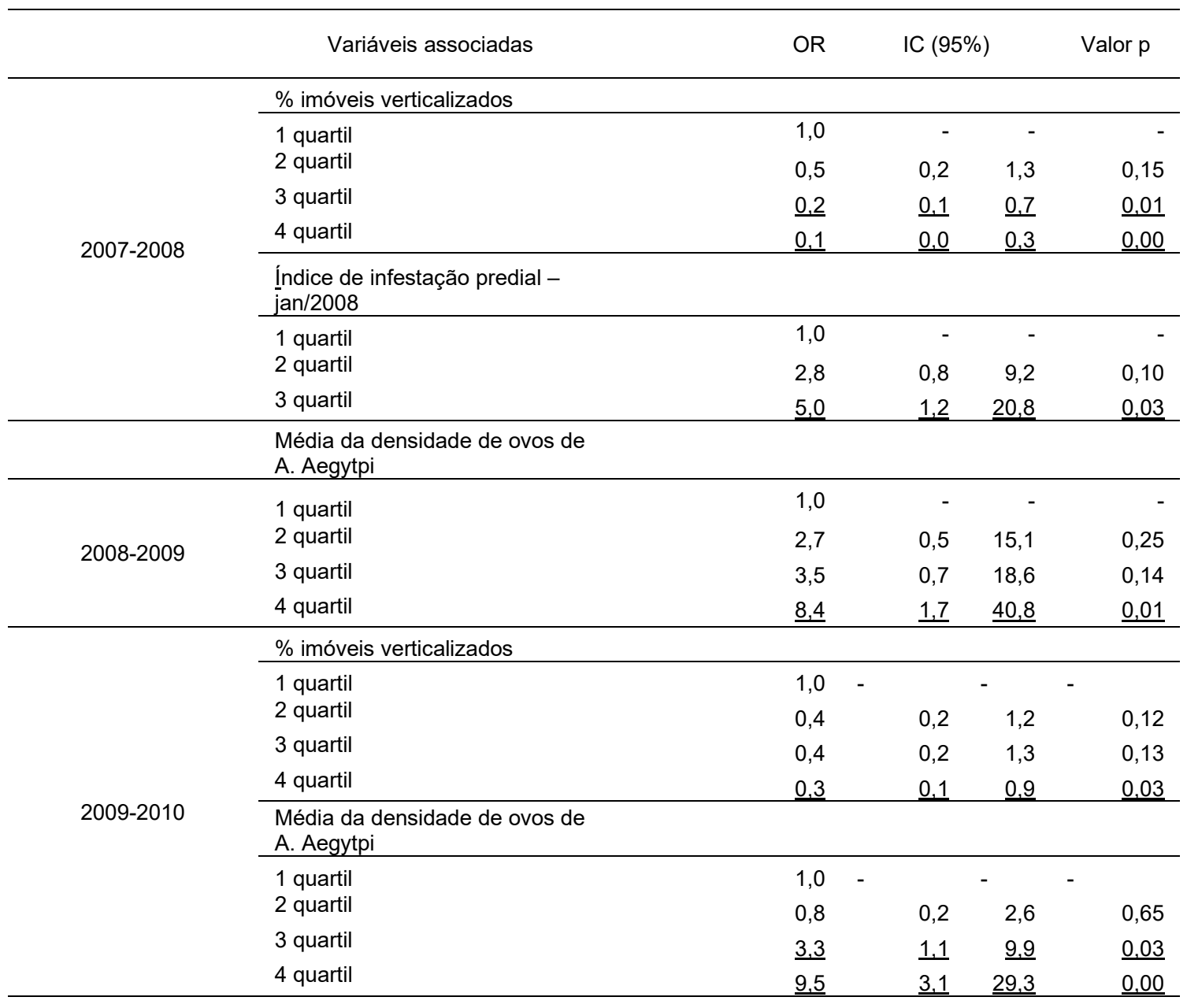

Fonte: Elaborado pelos autores

\section{DISCUSSÃO E CONCLUSÕES}

Este estudo possibilitou identificar clusters de transmissão de dengue em um capital de grande porte da região sudeste do Brasil, por meio da aplicação de estatística de varredura e de dados disponíveis e de fácil acesso por parte da vigilância municipal. Os principais fatores associados ao aumento de transmissão foram índice de infestação predial, densidade de ovos coletados por meio de ovitrampas e tipo de moradia.

O uso de áreas de abrangência facilita as estratégias de organização do serviço; entretanto, o uso de áreas menores, como setores censitários, podem revelar outras desigualdades intra-urbanas. Apesar disso, os achados deste estudo sugerem alta dependência espacial da doença devido às fortes associações e significância estatística encontrados.

A persistência de clusters na mesma região, nos três períodos analisados, levanta algumas hipóteses: sugere uma maior detecção de casos influenciada a maior cobertura de assistência a saúde, pode estar relacionada a condições socioambientais que tornam essa população mais vulnerável ou que ainda pode influenciar ou prejudicar as ações de controle vetorial nas referidas áreas. A associação entre a ocorrência de clusters e os indicadores entomológicos adotados no presente estudo reforçam a utilidade DOI: http://dx.doi.org/10.14393/Hygeia17057163 $\quad$ Hygeia $\quad$ v.17 $\quad$ p. 14 - 25, 2021 página 22 
Varredura espacial para identificação de áreas de risco epidêmico e fatores associados a dengue: experiência em Belo Horizonte, Minas Gerais
Amanda Priscila de Santana Cabral Silva

Dalva Maria de Assis

Lívia Carla Vinhal Frutuoso

Rodrigo Fabiano do Carmo Said

destes como preditores de surtos, como referido em outros estudos (GALLI, 2008; CORREA, 2008; TEIXEIRA, 2002; PONTES, 2000) e possibilita que medidas preventivas e estruturantes possam ser implementadas antes do período de alta transmissão.

A densidade de ovos associada aos clusters em dois dos três anos estudados, mostra sua maior predição comparado ao IIP, como também observado em estudos nas cidades de Salvador e Port of Spain (Braga, 2000; Rawlins, 1998). A ovitrampa é relatada como operacionalmente simples e economicamente viável para deteç̧ão do vetor. Desta forma, recomendamos que os programas de controle da dengue utilizem esse instrumento na rotina e adotem seus indicadores como complemento da vigilância da doença, a exemplo da experiência recente com o projeto ArboAlvo, que tem como objetivos desenvolver uma metodologia para estratificação do território em áreas de risco de transmissão de arboviroses urbanas, com base em parâmetros epidemiológicos, entomológicos, ambientais e sociodemográficos.

O presente estudo identificou relação inversa entre regiões com alta densidade de imóveis verticalizados e incidência de dengue, comportamento, relatado desde o ano 2000 em Belo Horizonte (CUNHA ET AL, 2008). Esse achado reforça a prática das visitas de agentes ambientais no térreo das edificações, o que teoricamente otimiza recursos. Entretanto, análise realizada por Glasser e colaboradores (2011) no litoral do estado de São Paulo, identificou altas taxas de infestação por A. aegypti tanto em áreas coletivas de prédios como em imóveis não residenciais de grande porte. A caracterização da problemática de diferentes imóveis precisa ser melhor avaliada pelos programas de controle, para melhor direcionamento das ações antivetoriais.

Como limitações deste estudo, estão o uso de dados secundários, o que pode levar a uma subnotificação de casos, a depender do nível de sensibilidade do Sistema. Erros de diagnóstico, problemas no acesso aos serviços de saúde e frequência de infecções assintomáticas são comumente associados à subnotificação, o que pode impactar no conhecimento da realidade local e no direcionamento das prioridades da gestão em saúde (MOL ET AL, 2020). Outra limitação seria o fato de considerar como unidade de análise a área de abrangência de residência, o que remete a uma transmissão exclusivamente domiciliar, embora essa transmissão possa ter ocorrido na escola, trabalho ou área de lazer. Por fim, como relatado em outros estudos, não considerarmos no cálculo dos coeficientes de incidência a redução de suscetíveis, a variedade de sorotipos circulantes e as migrações (PESSANHA, 2010; RESENDES, 2010; ALMEIDA, 2008; CUNHA, 2008), todavia inquéritos populacionais realizados no próprio município, referem que a transmissão da dengue depende, em sua maioria, de indicadores entomológicos, como os utilizados no presente estudo, do que de indicadores individuais.

A análise espacial como rotina do serviço é viável para ser implantada em outros municípios devido à disponibilidade de softwares livres e bases cartográficas do IBGE. O treinamento de técnicos é possível por meio de parcerias institucionais. A utilização e/ou criação de estratégias para o preenchimento automático dos campos de coordenadas geográficas dos formulários de notificação da dengue no Sistema de Informações de Agravos de Notificação é pertinente para operacionalização desta ferramenta.

A utilização de técnicas de geoprocessamento, a exemplo da varredura especial, com a inclusão de indicadores epidemiológicos e entomológicos, aliada à abordagens ecossistêmicas, capazes de identificar e agir sobre determinantes sociais e ambientais, podem contribuir sobremaneira na identificação de áreas de risco, ação fundamental para o planejamento de intervenções mais oportunas.

\section{AGRADECIMENTOS}

Agradecemos ao Dr. José Eduardo Marques Pessanha (in memorian), da Secretaria Municipal de Saúde de Belo Horizonte, por todo o incentive e apoio durante o planejamento e execução desse estudo. 
Varredura espacial para identificação de áreas de risco epidêmico e fatores associados a dengue: experiência em Belo Horizonte, Minas Gerais
Amanda Priscila de Santana Cabral Silva

Dalva Maria de Assis

Lívia Carla Vinhal Frutuoso

Rodrigo Fabiano do Carmo Said

\section{FINANCIAMENTO}

Este trabalho foi realizado durante o treinamento da autora principal no âmbito do Programa de Treinamento em Epidemiologia Aplicada aos Serviços do Sistema Único de Saúde (Episus), coordenado pela Secretaria de Vigilância em Saúde do Ministério da Saúde em Brasília-DF, Brasil. O estudo foi financiado sob a condição de sua autora principal enquanto bolsista do Conselho Nacional de Desenvolvimento Científico e Tecnológico (CNPq)/Ministério da Ciência e Tecnologia (MCT): Processo no 105776/2010-6.

\section{REFERÊNCIAS BIBLIOGRÁFICAS}

ALMEIDA, M.C.M. et al. Dinâmica intra-urbana das epidemias de dengue em Belo Horizonte, Minas Gerais, Brasil, 1996 - 2002. Cadernos de Saúde Pública, Rio de Janeiro, 24 (10), out, 2008. https://doi.org/10.1590/S0102-311X2008001000019

BRAGA, I.A. et al. Comparação entre pesquisa larvária e armadilha de oviposição, para detecção de Aedes aegypti. Revista da Sociedade Brasileira de Medicina Tropical, Uberaba, v. 33, n. 4, Aug. 2000 Disponível em: <http://www.scielo.br/scielo.php?script=sci_arttext\&pid=S003786822000000400003\&lng=en\&nrm=iso>. Acesso em 20 de janeiro de 2011. https://doi.org/10.1590/S0037-86822000000400003

CORREA, P.R.L. et al. Infestação pelo Aedes aegypti e ocorrência da dengue em Belo Horizonte, Minas Gerais. Revista de Saúde Pública [online]. 2005, vol.39, n.1, pp. 33-40. ISSN 0034-8910. https://doi.org/10.1590/S0034-89102005000100005

CUNHA, M.C.M. et al . Fatores associados à infecção pelo vírus do dengue no Município de Belo Horizonte, Estado de Minas Gerais, Brasil: características individuais e diferenças intra-urbanas. Epidemiologia e Serviços de Saúde, Brasília, v. 17, n. 3, set. 2008 . Disponível em <http://scielo.iec.pa.gov.br/scielo.php?script=sci_arttext\&pid=S1679-

49742008000300007\&lng=pt\&nrm=iso>. Acesso em 20 de janeiro de 2011. https://doi.org/10.5123/S1679-49742008000300007

GALLI, B.; CHIARAVALLOTI NETO, F.. Modelo de risco tempo-espacial para identificação de áreas de risco para ocorrência de dengue. Revista de Saúde Pública, São Paulo, v. 42, n. 4, Aug. 2008. Disponível em <http://www.scielo.br/scielo.php?script=sci_arttext\&pid=S0034$89102008000400011 \&$ Ing=en\&nrm=iso>. Acesso em 20 de dezembro de 2010. https://doi.org/10.1590/S0034-89102008000400011

GLASSER, C.M. et al . Comportamento de formas imaturas de Aedes aegypti, no litoral do Estado de São Paulo. Rev. Soc. Bras. Med. Trop., Uberaba, v. 44, n. 3, p. 349-355, June 2011 . Disponível em <http://www.scielo.br/scielo.php?script=sci_arttext\&pid=S0037-86822011000300018\&lng=en\&nrm=iso>.

INSTITUTO BRASILEIRO DE GEOGRAFIA E ESTATÍSTICA (IBGE). Censo demográfico 2010. Características da população e dos domicílios: resultado do universo Rio de Janeiro: IBGE; 2010. https://doi.org/10.1590/S0037-86822011005000042

KULLDORFF, M. SaTScan user guide. Versão fevereiro, 2015. Disponível em <http://www.satscan.org/references.html>. Acesso em: 15 ago. 2020.

MARQUES, C. A.; SIQUEIRA, M. M.; PORTUGAL, F. B.. Avaliação da não completude das notificações compulsórias de dengue registradas por município de pequeno porte no Brasil. Ciência \& Saúde Coletiva, Rio de Janeiro , v. 25, n. 3, p. 891-900, Mar. 2020 . Disponível em: <http://www.scielo.br/scielo.php?script=sci_arttext\&pid=S1413-81232020000300891\&lng=en\&nrm=iso>.

Acesso em: 07 Set. 2020. https://doi.org/10.1590/1413-81232020253.16162018

MINISTÉRIO DA SAÚDE. Diretrizes nacionais para prevenção e controle de epidemias de dengue. Brasília, 2009. 160p - Série A. normas e Manuais Técnicos. 
Varredura espacial para identificação de áreas de risco epidêmico e fatores associados a dengue: experiência em Belo Horizonte, Minas Gerais
Amanda Priscila de Santana Cabral Silva

Dalva Maria de Assis

Lívia Carla Vinhal Frutuoso Rodrigo Fabiano do Carmo Said

MINISTÉRIO DA SAÚDE. Secretaria de Vigilância em Saúde. Informe Epidemiológico da Dengue Análise de situação e tendências - Até a semana 26 de 2010. Nota técnica, 2010. Disponível em: http://portal.saude.gov.br/portal/arquivos/pdf/informe_dengue_se_26_final_11_8_10.pdf

MOL MPG, Queiroz JTM, Gomes J, Heller L. Gestão adequada de resíduos sólidos como fator de proteção na ocorrência da dengue. Rev Panam Salud Publica. 2020;44:e22. https://doi.org/10.26633/RPSP.2020.22

PAVARINI, S.C.I. et al . Sistema de informações geográficas para a gestão de programas municipais de cuidado a idosos. Texto contexto - enfermagem, Florianópolis , v. 17, n. 1, p. 17-25, Mar. 2008. Available from <http://www.scielo.br/scielo.php?script=sci_arttext\&pid=S010407072008000100002\&lng=en\&nrm=iso>. access on 09 Aug. 2020. https://doi.org/10.1590/S0104$\underline{07072008000100002}$

PESSANHA, J.E.M. et al . Dengue em três distritos sanitários de Belo Horizonte, Brasil: inquérito soroepidemiológico de base populacional, 2006 a 2007. Revista Panamerica de Saúde Pública, Washington, $\quad$ v. 27, n. 4, Apr. $2010 \quad$. Disponível em $<$ http://www.scielosp.org/scielo.php?script=sci_arttext\&pid=S1020-

49892010000400003\&lng=en\&nrm=iso>. Acesso em 20 jan. 2010. https://doi.org/10.1590/S1020$\underline{49892010000400003}$

PONTES, R.J. et al. Vector densities that potentiate dengue outbreaks in a Brazilian city. American Journal Tropical Medicine and Hygiene, v.62, p.378-83, 2000. Disponível em: http://www.ncbi.nlm.nih.gov/pubmed/11037781 Acesso em 30 de dezembro de 2010. https://doi.org/10.4269/ajtmh.2000.62.378

RAWLINS S.C., MARTINEZ R., WILTSHIRE, L.G.A. Comparison of surveillance systems for the dengue vector Aedes aegypti in Port of Spain, Trinidad. Journal of the American Mosquito Control Association 14:131-136, 1998. Disponível em: http://www.ncbi.nlm.nih.gov/pubmed/9673912. Acesso em 15 de janeiro de 2011.

RESENDES, A.P.C. et al . Determinação de áreas prioritárias para ações de controle da dengue. Revista de Saúde Pública, São Paulo, v. 44, n. 2, Apr. 2010 . Disponível em: <http://www.scielo.br/scielo.php?script=sci_arttext\&pid=S0034-89102010000200007\&lng=en\&nrm=iso>. Acesso em: 30 nov. 2010. https://doi.org/10.1590/S0034-89102010000200007

SOUZA, A.C. et al. Dengue. Boletim Epidemiológico. 2019 set; 50(n.esp.):12-13. (Número especial: Vigilância em Saúde no Brasil 2003|2019: da criação da Secretaria de Vigilância em Saúde aos dias atuais). Disponível em: http://www.saude.gov.br/ boletins-epidemiologicos. Acesso em 15 de Agosto de 2020.

SOUZA, R.C.F. et al. Viver próximo à saúde em Belo Horizonte. Caderno Metropolitano, São Paulo, v. 18, n. 36, pp. 325-344, jul 2016. Disponível em: < https://www.scielo.br/pdf/cm/v18n36/2236-9996-cm-1836-0325.pdf>. Acesso em 10 de Agosto de 2020. https://doi.org/10.1590/2236-9996.2016-3601

TEIXEIRA M.G. et al. Cairncross S. Dynamics of dengue virus circulation: a silent epidemic in a complex urban area. Tropical Medicine and International Health. 2002;7(9):757-62. Disponível em: <http://iah.iec.pa.gov.br/iah/fulltext/pc/artigos/2002/TropMedlnterHeal\%20-\%20v7n9p757762\%202002.pdf > Acesso em 27 dez. 2010. https://doi.org/10.1046/j.1365-3156.2002.00930.x

VERONESI, R; FOCACCIA, R. Dengue. Tratado de infectologia: v.1. Säo Paulo, Atheneu, 2015. 